\title{
A 10-year-old boy with dyspnea and hypoxia: abernathy malformation masquerading as pulmonary arteriovenous fistula
}

\author{
Lijian Xie, Yun Li, Xunwei Jiang, Jian Zhao and Tingting Xiao* (D)
}

\begin{abstract}
Background: Abernethy malformation is an extremely rare congenital malformation characterised by an extrahepatic portosystemic shunt. Children with Abernathy malformation can develop hepatopulmonary syndrome (HPS) with pulmonary arteriovenous fistulas (PAVF) or pulmonary hypertension. PAVF manifests as central cyanosis with effort intolerance. We report a case of PAVF in a Ten-year-old Boy. Persistent symptoms identified Abernathy malformation as the cause of progressive symptoms and current understanding of this rare malformation is reviewed.
\end{abstract}

Case presentation: A case of 10-year-old boy with Abernethy malformation complicated with HPS initially managed as PAVF was presented. Selective lung angiography showed a typical diffuse reticular pattern on right lower lung, which suggested PAVF. However, cyanosis was not improved post transcatheter coil embolization. Then, liver disease was considered although the patient had normal aspartate aminotransferase and alanine aminotransferase. The significantly elevated serum ammonia was attracted our attention. Abdominal computed tomography also exhibited enlarged main portal vein (MPV), cirsoid spleen vein, and superior mesenteric vein (SMV). Angiography with direct opacification of the SMV with a catheter coming from the inferior vena cava (IVC) and going to the SMV via the shunt vessel (SHUNT) between the MPV and IVC. Occlusion the IVC with an inflated balloon, injection of contrast medium via a catheter placed in the SMV, MPV was showed and absence of intrahepatic branches. Abernethy malformation IB type is finally confirmed.

Conclusions: Abernethy malformation is an unusual cause for development of PAVF and cyanosis in children. Clinicians must be suspicious of Abernethy malformation complicated with HPS. If patients have abnormal serum ammonia and enlarged MPV in abdominal $C T$, cathether angiography should be done to rule out Abernethy malformation.

Keywords: Abernethy malformation, Hepatopulmonary syndrome, Pulmonary arteriovenous fistula

\section{Background}

Abernethy malformation is an extremely rare congenital malformation characterised by an extrahepatic portosystemic shunt. It was first reported by John Abernethy and it is named Abernethy malformation [1]. Until now, more than 300 cases have been reported with a literature review, and most patients were female and less than 18 years old [2, 3]. Abernethy malformation can be

* Correspondence: ttxiao2017@163.com

Department of Cardiology, Shanghai Children's Hospital, Shanghai Jiaotong University, No. 355 Luding Road, Shanghai 200062, China classified into two types. Type I is defined by an absence of intrahepatic portal veins, and lack of liver perfusion with portal blood. Type I anomaly may be further divided into subtype $\mathrm{A}$ and $\mathrm{B}$, defined as superior mesenteric and splenic veins draining separately into the inferior vena cava (IVC) in type IA or draining from a common trunk in type IB [4]. Type II anomaly consists of a hypoplastic portal vein supplying the liver through side-to-side anastomosis, and a larger branch that drains directly into the IVC, which means hypoplastic

(c) The Author(s). 2019 Open Access This article is distributed under the terms of the Creative Commons Attribution 4.0 International License (http://creativecommons.org/licenses/by/4.0/), which permits unrestricted use, distribution, and reproduction in any medium, provided you give appropriate credit to the original author(s) and the source, provide a link to the Creative Commons license, and indicate if changes were made. The Creative Commons Public Domain Dedication waiver (http://creativecommons.org/publicdomain/zero/1.0/) applies to the data made available in this article, unless otherwise stated. 
intrahepatic portal vein with some degree of portal flow into the liver [5].

The clinical manifestations of Abernethy malformation are highly variable and can be divided into 3 types: (1)asymptomatic, (2)symptoms due to the abnormal liver development such as hepatic encephalopathy or multiple liver nodules/tumors, and (3)shunt related symptoms such as pulmonary hypertension or hepatopulmonary syndrome (HPS) [4, 6-8]. HPS is characterized by the presence of dyspnea and hypoxia in patients with liver diseases. Pulmonary arteriovenous fistula (PAVF) is a rare complication of Abernethy malformation, which is occurred secondary to liver failure or bypassing of the liver by portosystemic shunt [9]. PAVF is clinically and radiologically divided into simple and complex type and commonly associated with hereditary hemorrhagic telangiectasia (HHT) [10]. PAVF is not easily diagnosed routinely, due to its rarity and its unspecific findings on routine examinations [11]. Chest computed tomography (CT) and selective lung angiography are helpful to diagnose PAVF. If the patient's clinical presentation is hypoxia caused by PAVF, its primary disease (Liver disease or Abernethy malformation) is easily to be likely to be overlooked in the initial evaluation. .

Here, a case of 10-year-old boy with Abernethy malformation complicated with HPS who is initially managed as PAVF.

\section{Case presentation}

A 10-year-old Chinese boy, with a height of $152 \mathrm{~cm}$ and weighing $35 \mathrm{~kg}$, was presented with 4-year history of cyanosis and dyspnea on exertion. Physical examination on admission revealed a central cyanosis and digital clubbing with a resting pulse oximetry $\left(\mathrm{SpO}_{2}\right)$ of $75 \%$ on room air. Chest and cardiac examination results were unremarkable. His abdominal examination showed situs solitus and no hepatomegaly. He had mild mental retardation, however, no evidence of encephalopathy. His laboratory test showed an elevated hemoglobin level of $16.5 \mathrm{~g} / \mathrm{L}$, a normal liver enzyme enzyme profile with aspartate aminotransferase $16 \mathrm{U} / \mathrm{L}$, alanine aminotransferase $20 \mathrm{U} / \mathrm{L}$. Direct bilirubin was $4 \mu \mathrm{mol} / \mathrm{L}$ (normal range 0 to $6.8 \mu \mathrm{mol} / \mathrm{L}$ ) and albumin was $40.8 \mathrm{~g} / \mathrm{L}$ (normal range 38 to $54 \mathrm{~g} / \mathrm{L}$ ). Chest X-ray, electrocardiogram and echocardiogram results were unremarkable. Chest $\mathrm{CT}$ showed diffuse pulmonary hypervascularization. Hence, diffuse PAVF was suspected. A right cardiac catheterization was performed, which showed a normal pulmonary artery pressure. Selective lung angiography showed immediate opacification of the left atrium, and typical diffuse reticular vessel pattern on right lower lung, which suggested PAVF (Fig. 1). Transcatheter coil embolization for PAVF of 7 micro coils was performed, however, pulmonary arteriovenous shunt was still existing post occlusion (Fig. 1). And, symptoms of cyanosis and dyspnea were not improved.

So, we began to suspect our original diagnosis of PAVF and liver disease was considered. We found the serum ammonia was elevated to $82 \mu \mathrm{mol} / \mathrm{L}$ (normal range from 16 to $60 \mu \mathrm{mol} / \mathrm{L}$ ). The elevated serum ammonia was attracted our attention. Furthermore, abdominal contrast enhanced CT showed the main portal vein (MPV) was enlarged, spleen vein (SV) and superior mesenteric vein (SMV) and its branches were circuity expansion. Congenital extrahepatic portosystemic shunt was considered. A selective cathether angiography was subsequently performed, and the results confirmed it was Abernethy malformation IB type. Angiography with direct opacification of the SMV with a catheter coming from the IVC and going to the
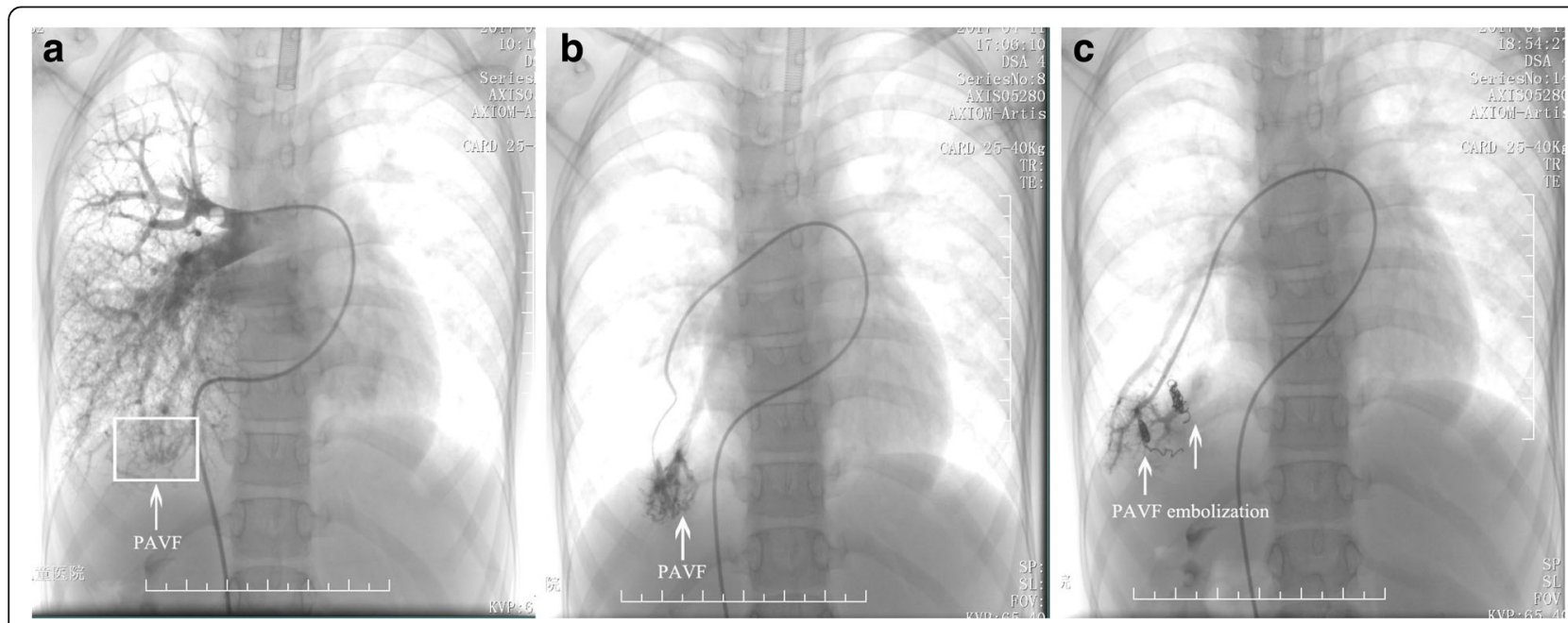

Fig. 1 a A selective right pulmonary arteriogram showed diffuse reticular pattern on right lower lung, which suggested pulmonary arteriovenous fistula (PAVF). b Right lower pulmonary arteriogram with micro catheter showed diffuse and distorted PAVF. c After the coil embolization, pulmonary arteriovenous shunt was significantly reduced 
SMV via the shunt vessel (SHUNT) between the MPV and IVC (Fig. 2). Occlusion the IVC with an inflated balloon, injection of contrast medium via a catheter placed in the SMV, MPV was showed and no intrahepatic branches could be opacified (Fig. 2). Finally, the investigatory features were consistent with the diagnosis of type IB Abernethy malformation, and the child is now scheduled for Liver transplantation.

\section{Discussion and conclusions}

Although overall a rare malformation, Abernethy malformation is being diagnosed more frequently with the advances in imaging techniques. Contrast CT scan and Three-dimensional (3D) vessel reconstruction could confirm Abernethy malformation diagnosis in children and adults [12-14]. The 3D reconstruction of blood vessels is very useful for demonstrating extrahepatic portocaval shunts in patients who are suspected Abernethy malformation [15]. Moreover, an experienced sonographer could detect side-to-side communication between the MPV and IVC in subcostal window [16, 17]. Anyway, selective cathether angiography in the MPV is a golden diagnostic standard.

Here we reported a 10-year-old male Abernethy malformation, which exhibited cyanosis and dyspnea. Firstly, selective lung angiography showed the appearance of dilated distal pulmonary arteries and a classic reticular pattern suggestive of PAVF. However, $\mathrm{SpO}_{2}$ and cyanosis were not improved post transcatheter coil embolization. We reviewed the boy's clinical, biochemical and radiological data again. The elevated serum ammonia caused our attention. Abdominal CT also showed enlarged MPV, cirsoid SV and SMV. Finally, Abernethy malformation IB type is confirmed by cathether angiography. PAVF is secondary to Abernethy malformation complicated with HPS.

HPS is a triad of liver diseases, arterial hypoxemia and pulmonary vascular dilatation. Although HPS typically develops in the presence of cirrhosis or portal hypertension, it may also occur in the absence of parenchymal liver disease in association with portosystemic shunting. To our knowledge, Abernethy malformation with HPS was first reported in a 3-year-old girl with polysplenia syndrome in 1993 [18]. Until now, HPS has been reported in at least 20 children with Abernethy malformation [19]. Three hypothesis accounting for the etiology have been proposed, that is, elevated endothelin-1 circulating in the whole body which up-regulates nitric oxide $(\mathrm{NO})$ production in the lungs by continuously stimulating the NO synthase, hepatic products necessary for pulmonary vasomotor control are decreased by liver dysfunction or hepatic venous flow reduction, (3)translocation of gut bacteria activating alveolar macrophages results in increase in inducible NO synthase [13]. In a word, HPS is believed to be attributed to the exposure of the pulmonary vascular bed to vasoactive mediators, which derived from the intestinal tract entering the systemic circulation without being metabolized in the liver [3]. So, PAVF is occurred secondary to liver failure or bypassing of the liver by portosystemic shunt [5].

Treatment of congenital malformations of the portal system depends on the type of Abernethy malformation, the presenting symptoms, complications and comorbidity.
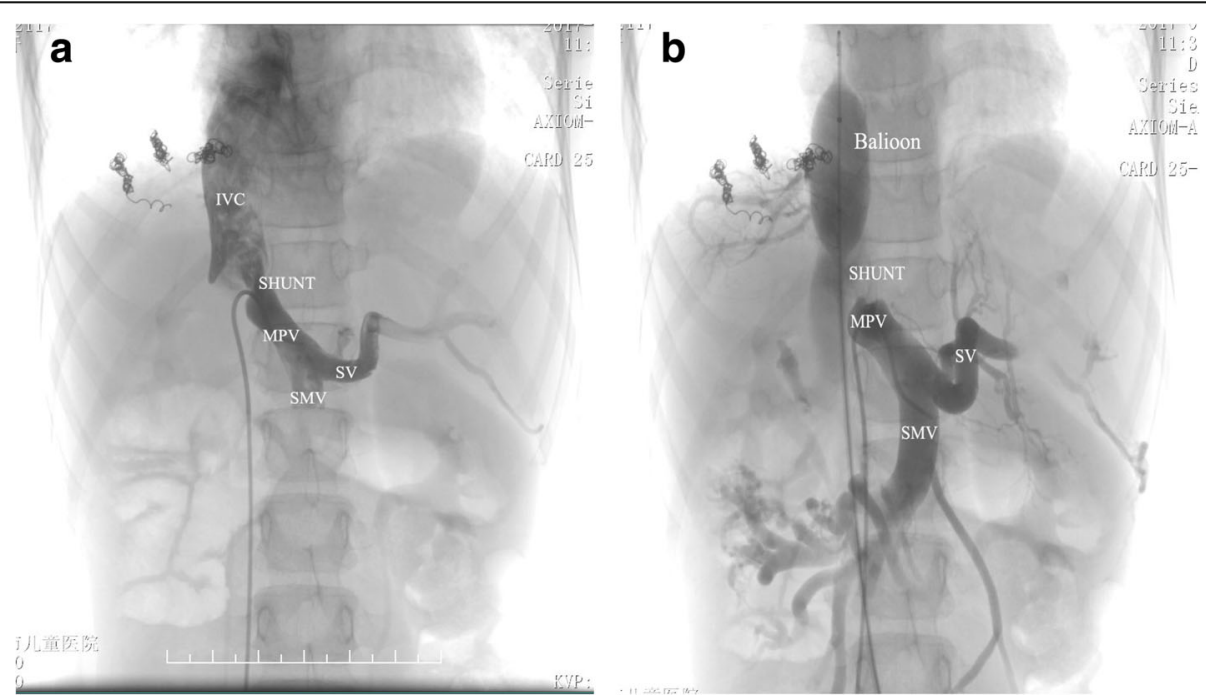

Fig. 2 An angiogram showed side to side communication between the main portal vein (MPV) and the inferior vena cava (IVC) in our case. a Angiography with direct opacification of the superior mesenteric vein (SMV) with a catheter coming from the IVC and going to the SMV via the shunt vessel (SHUNT) between the MPV and IVC. SMV and splenic vein (SV) are draining from a common trunk. b Occlusion the IVC with a inflated balloon, injection of contrast medium via a catheter placed in the SMV, MPV was showed and no intrahepatic branches were showed. So, our case is confirmed as Abernethy malformation IB type 
Treatment may vary from surgical correction or transcatheter occlusion of the shunt to even liver transplantation $[19,20]$. For pediatric patients with extrahepatic or intrahepatic portosystemic shunt, the appropriate time of therapy has not been confirmed [21]. It is proposed that even in the absence of overt symptoms, early intervention prevents pulmonary complications and neurodevelopmental delay could be avoided [22]. The clear clinical intervention indications are encephalopathy, HPS, porto-pulmonary hypertension, liver nodules regenerating, and increasing shunt size if the patient is fit to tolerate any invasive procedure [21]. The choice of transcatheter or surgical approach depends on the classification of the malformation as type I or II. The selection of percutaneous embolic materials is depending on shunt size, which included coils, detachable balloons, and vascular plugs [21]. A temporary balloon occlusion test is very important to evaluate the portal vein pressure during the intervention procedure. Surgical ligation is the another method in cases with large shunts with risk of materials migration during embolization or post embolization failure [21].

Liver transplantation has been performed for Abernethy malformation type I. Given the lack of an apparent intrahepatic portal venous system in type I disease, liver transplantation is a definitive therapy. Sakamoto et al. have reviewed 34 patients treated by liver transplantation for extrahepatic shunts. Good outcomes were reported, with 31 patients alive $(91.2 \%)$ at a median follow-up of 18 months [23, 24]. Malignant tumors have been reported to occur in Abernethy malformation patients in the absence of liver dysfunction and cirrhosis (4\% of all cases) [25]. Indications for liver transplant remain poorly defined except for hepatocellular carcinoma cases [23, 24].

Exploiting phenotypic plasticity for an 11-year-old boy with HPS secondary to type I Abernethy malformatio was firstly reported by Kuo et al. [26]. It is a new treatment for Abernethy malformation, which included 3-staged endovascular method. Stage 1, portal venous outflow tract establishment by creating a transjugular intrahepatic portosystemic shunt (TIPS), then a second covered stent was placed in his IVC to block the portacaval fistula. Stage 2, 6 weeks later, intrahepatic portal venous network was developed. The TIPS stent was reduced. Stage 3, next 6 weeks later, normal left and right portal veins and normal-sized intrahepatic portal veins were confirmed by venography The remaining TIPS was completely blocked with Amplatzer Vascular Plug and/or embolization coils [26]. The patient totally remains free of HPS stigmata 2 years later. This case provides a new therapy strategy in patient with Abernethy malformation and HPS.
In conclusion, via this case, we make a point that Abernethy malformation is an unusual cause for development of PAVF and cyanosis in children Clinicians must be suspicious of Abernethy malformation complicated with HPS as abnormally elevated serum ammonia levels and enlarged portal veins with demonstration of portosystemic fisulas on imaging are other diagnostic clues.

\section{Abbreviations}

HPS: Hepatopulmonary syndrome; IVC: Inferior vena cava; MPV: Main portal vein; NO: Nitric oxide; PAVF: Pulmonary arteriovenous fistula; SMV: Superior mesenteric vein; SV: Spleen vein

\section{Acknowledgements}

Not applicable.

\section{Funding}

This work is supported by Research Fund for Shanghai Healthy Committee (grant number: 201540099), Shanghai Talents Developing Committee (grant number: 201615), Shanghai science and technology committee (grant number: 17140902100) and Shanghai Jiaotong University medical technology crossing project (YG2016ZD05). No benefits in any form have been or will be received from a commercial organization directly or indirectly.

\section{Availability of data and materials}

All data generated or analyzed during this study are included in this published article and its Additional files.

\section{Authors' contributions}

LJX and TTX designed and operated the project. JZ, YL and XWJ provided the radiological analyses. $L X$ wrote the manuscript with input from JZ, YL and XWJ. All authors read and approved the final manuscript.

Ethics approval and consent to participate

The ethical approval and documentation for this case report were waived with approval from the Institutional Review Board of Shanghai Children's Hospital.

\section{Consent for publication}

Written informed consent was obtained from the patient's parents for publication of this case report including clinical data and any accompanying images.

\section{Competing interests}

The authors declare that they have no competing interests.

\section{Publisher's Note}

Springer Nature remains neutral with regard to jurisdictional claims in published maps and institutional affiliations.

Received: 5 April 2018 Accepted: 29 January 2019

Published online: 11 February 2019

References

1. Abernethy J.J Banks. Account of two instances of uncommon formation in the viscera of the human body. Phil Trans R Soc Lond 1793;83:59-66.

2. Hao $Y$, Hong $X$, Zhao $X$. Congenital absence of the portal vein associated with focal nodular hyperplasia of the liver and congenital heart disease (Abernethy malformation): a case report and literature review. Oncol Lett. 2015;9(2):695-700.

3. Sokollik C, Bandsma RH, Gana JC, van den Heuvel M, Ling SC. Congenital portosystemic shunt: characterization of a multisystem disease. J Pediatr Gastroenterol Nutr. 2013;56(6):675-81.

4. Witters P, Maleux G, George C, Delcroix M, Hoffman I, Gewillig M, Verslype C, Monbaliu D, Aerts R, Pirenne J, Van Steenbergen W, Nevens F, Fevery J, 
Cassiman D. Congenital veno-venous malformations of the liver: widely variable clinical presentations. J Gastroenterol Hepatol. 2008;23:e390-4.

5. Morgan G, Superina R. Congenital absence of the portal vein: two cases and a proposed classification system for portasystemic vascular anomalies. J Pediatr Surg. 1994;29:1239-41.

6. Osorio MJ, Bonow A, Bond GJ, et al. Abernethy malformation complicated by hepatopulmonary syndrome and a liver mass successfully treated by liver transplantation. Pediatr Transplant. 2011;15:E149-51.

7. De Gaetano AM, Gui B, Macis G, Manfredi R, Di Stasi C. Congenital absence of the portal vein associated with focal nodular hyperplasia in the liver in an adult woman: imaging and review of the literature. Abdom Imaging. 2004;29:455-9

8. Pichon N, Maisonnette F, Pichon-Lefievre F, et al. Hepatocarcinomawith congenital agenesis of the portal vein. Jpn J Clin Oncol. 2003;33:314-6.

9. Alvarez AE, Ribeiro AF, Hessel G, Baracat J, Ribeiro JD. Abernethy malformation: one of the etiologies of hepatopulmonary syndrome. Pediatr Pulmonol. 2002;34:391-4.

10. Gill SS, Roddie ME, Shovlin CL, Jackson JE. Pulmonary arteriovenous malformations and their mimics. Clin Radiol. 2015;70:96-110.

11. Ahn S, Han J, Kim HK, Kim TS. Pulmonary arteriovenous fistula: clinical and histologic Spectrum of four cases. J Pathol Transl Med. 2016;50(5):390-3.

12. Mi XX, Li XG, Wang ZR, Lin L, Xu CH, Shi JP. Abernethy malformation associated with Caroli's syndrome in a patient with a PKHD1 mutation: a case report. Diagn Pathol. 2017;12(61):1-6.

13. Sahu MK, Bisoi AK, Chander NC, Agarwala S, Chauhan S. Abernethy syndrome, a rare cause of hypoxemia: a case report. Ann Pediatr Cardiol. 2015;8(1):64-6.

14. Soni N, Ambesh P, Sunil K, Jena M. Abernethy malformation with inferior vena cava stenosis: a cryptic cause of cyanosis. Indian Heart J. 2015;67(6):543-5.

15. Konstas AA, Digumarthy SR, Avery LL, Wallace KL, Lisovsky M, Misdraji J, Hahn PF. Congenital portosystemic shunts: imaging findings and clinical presentations in 11 patients. Eur J Radiol. 2011;80(2):175-81.

16. Venkateshwaran S, Krishnamoorthy KM, Sivasankaran S. Percutaneous device closure of Abernethy malformation--a treatable cause of hepatopulmonary syndrome. Catheter Cardiovasc Interv. 2014;83(6):968-70.

17. Chira Rl, Calauz A, Manole S, Valean S, Mircea PA. Unusual discovery after an examination for abdominal pain: Abernethy $1 \mathrm{~b}$ malformation and liver Adenomatosis. A case report. J Gastrointestin Liver Dis. 2017;26(1):85-8.

18. Papagiannis J, Kanter RJ, Effman EL, Pratt PC, Marcille R, Browning IB 3rd, Armstrong BE. Polysplenia with pulmonary arteriovenous malformations. Pediatr Cardiol. 1993;14(2):127-9

19. Fu L, Wang Q, Wu J, Guo Y, Huang M, Liu T, Chen Q, Li F. Congenital extrahepatic portosystemic shunt: an underdiagnosed but treatable cause of hepatopulmonary syndrome. Eur J Pediatr. 2016;175(2):195-201.

20. Singhal A, Srivastava A, Goyal N, Vij V, Wadhawan M, Bera M, Gupta S. Successful living donor liver transplant in a child with Abernethy malformation with biliary atresia, ventricular septal defect and intrapulmonary shunting. Pediatr Transplant. 2009;13:1041-7.

21. Papamichail M, Pizanias M, Heaton N. Congenital portosystemic venous shunt. Eur J Pediatr. 2018;177:285-94.

22. PapamichailM AA, Quaglia A, Karani J, Heaton N. Liver resection for the treatment of a congenital intrahepatic portosystemic venous shunt. Hepatobiliary Pancreat Dis Int. 2016;15(3):329-33.

23. Sakamoto S, Shigeta T, Fukuda A, Tanaka H, Nakazawa A, Nosaka S, et al. The role of liver transplantation for congenital extrahepatic portosystemic shunt. Transplantation. 2012;93(12):1282-7.

24. Sakamoto S, Kasahara M, Shigeta T, Fukuda A, Kakiuchi T, Miyasaka M, et al. Living donor liver transplantation for multiple intrahepatic portosystemic shunts after involution of infantile hepatic hemangiomas. J Pediatr Surg. 2011:46(6):1288-91.

25. Lautz TB, Shah SA, Superina RA. Hepatoblastoma in children with congenital portosystemic shunts. J Pediatr Gastroenterol Nutr. 2016;62(4):542-5

26. Kuo MD, Miller FJ, Lavine JE, Peterson M, Finch M. Exploiting phenotypic plasticity for the treatment of Hepatopulmonary shunting in Abernethy malformation. J Vasc Interv Radiol. 2010;21:917-22.

\section{Ready to submit your research? Choose BMC and benefit from:}

- fast, convenient online submission

- thorough peer review by experienced researchers in your field

- rapid publication on acceptance

- support for research data, including large and complex data types

- gold Open Access which fosters wider collaboration and increased citations

- maximum visibility for your research: over $100 \mathrm{M}$ website views per year

At BMC, research is always in progress.

Learn more biomedcentral.com/submissions 